\title{
Metric for gradient renormalization group flow of the worldsheet sigma model beyond first order
}

\author{
T. Oliynyk, ${ }^{1, *}$ V. Suneeta, ${ }^{2, \dagger}$ and E. Woolgar ${ }^{3, *}$ \\ ${ }^{1}$ Max-Planck-Institut für Gravitationsphysik (Albert Einstein Institute), Am Mühlenberg 1, D-14476 Potsdam, Germany \\ ${ }^{2}$ Department of Mathematics and Statistics, University of New Brunswick, Fredericton, New Brunswick, Canada E3B 5A3 \\ ${ }^{3}$ Department of Mathematical and Statistical Sciences, University of Alberta, Edmonton, Alberta, Canada T6G $2 G 1$
}

(Received 6 May 2007; published 2 August 2007)

Tseytlin has recently proposed that an action functional exists whose gradient generates to all orders in perturbation theory the renormalization group (RG) flow of the target space metric in the worldsheet sigma model. The gradient is defined with respect to a metric on the space of coupling constants which is explicitly known only to leading order in perturbation theory, but at that order is positive semidefinite, as follows from Perelman's work on the Ricci flow. This gives rise to a monotonicity formula for the flow which is expected to fail only if the beta function perturbation series fails to converge, which can happen if curvatures or their derivatives grow large. We test the validity of the monotonicity formula at next-toleading order in perturbation theory by explicitly computing the second-order terms in the metric on the space of coupling constants. At this order, this metric is found not to be positive semidefinite. In situations where this might spoil monotonicity, derivatives of curvature become large enough for higher-order perturbative corrections to be significant.

DOI: 10.1103/PhysRevD.76.045001

PACS numbers: $11.10 . \mathrm{Hi}$

\section{INTRODUCTION}

It has been known for quite a long time that the renormalization group (RG) flow of 2-dimensional nonlinear sigma models, computed to first order in the loop expansion and neglecting all but gravity, is a gradient flow generated by the Einstein-Hilbert action. The first-order RG flow [1] is the Ricci flow, which can be written as

$$
\frac{\partial g_{i j}}{\partial t}=-\alpha^{\prime} R_{i j}=\operatorname{Grad}\left[\alpha^{\prime} \int_{M} R d V\right] .
$$

Here we take $g_{i j}$ to be a Riemannian metric, $d V$ to be the metric volume element, $R=g^{i j} R_{i j}$ to be the scalar curvature of the metric, $t$ to be the logarithm of the renormalization scale, and $\alpha^{\prime}>0$ to be a constant, ${ }^{1}$ the string length squared, which serves as an expansion parameter in the sigma model context.

The gradient here is on the "space of coupling constants," which we take to be the space whose points represent positive symmetric 2-tensor fields on a manifold $M$. The inner product of the gradient vector with another vector is a directional derivative which, in the present context, is the first variational derivative of an "action functional" or potential that generates the gradient flow (for greater detail, see Sec. IV).

\footnotetext{
*todd.oliynyk@aie.mpg.de

Address after 1 June 2007: School of Mathematical Sciences, Monash University, Vic 3800, Australia.

'suneeta@math.unb.ca

Address after 1 July 2007: Department of Mathematical and Statistical Sciences, University of Alberta, Edmonton AB, Canada T6G 2G1.

*ewoolgar@math.ualberta.ca

${ }^{1}$ This constant is usually set equal to 2 in the mathematics literature.
}

Now the variational derivative of the Einstein-Hilbert action

$$
S_{\mathrm{EH}}:=\int_{M} R d V
$$

on a closed manifold $M$ (so there are no boundary terms) ${ }^{2}$ in the direction $\frac{\partial g_{i j}}{\partial s}$ gives the very familiar result:

$$
\begin{aligned}
\frac{d S_{\mathrm{EH}}}{d s} & =-\int_{M}\left(R^{i j}-\frac{1}{2} g^{i j} R\right) \frac{\partial g_{i j}}{\partial s} d V \\
& =-\int_{M} R_{i j}\left(g^{i k} g^{j l}-\frac{1}{2} g^{i j} g^{k l}\right) \frac{\partial g_{k l}}{\partial s} d V .
\end{aligned}
$$

If the metric were $\langle u, v\rangle=\int_{M} u_{i j} v_{k l} g^{i k} g^{j l} d V$ then the gradient would be the negative of the Einstein tensor, but if the metric is

$$
\langle u, v\rangle:=\int_{M} u_{i j}\left(g^{i k} g^{j l}-\frac{1}{2} g^{i j} g^{k l}\right) v_{k l} d V,
$$

then the gradient is indeed the negative of the Ricci tensor [2], verifying the second equality in (1.1), and giving the formula

$$
\frac{d S_{\mathrm{EH}}}{d t}=\alpha^{2}\langle\text { Ric, Ric }\rangle
$$

for the derivative of the action along the flow. If the metric $\langle\cdot, \cdot\rangle$ were positive semidefinite, this formula would show that the action would increase monotonically along the flow, but obviously this metric is not of definite sign. As a result, the gradient can in principle change between being "timelike" and being "spacelike" according to whether the trace or tracefree part of the Ricci tensor dominates. Along any flow for which such a change occurs, the

\footnotetext{
${ }^{2}$ Throughout we take $(M, g)$ to be a closed Riemannian manifold.
} 
Einstein-Hilbert action will not be a monotonic function of the flow parameter.

The apparent lack of a monotonicity formula along the RG flow is surprising in view of the Zamolodchikov $C$-theorem [3], which guarantees a monotonic quantity along RG flow for a 2-dimensional unitary quantum field theory with a finite number of couplings [as opposed to the current case, where the coupling constants, usually found by expanding $g_{i j}(x)$ around a point $x_{0} \in M$, are infinite in number]. For a discussion of the problems associated with generalizing the $C$-theorem to the worldsheet sigma model (on a curved worldsheet), we refer the reader to the summary in [4].

There is, however, another approach which does yield a monotonicity formula for first-order RG flow and possibly beyond. In his celebrated work on Ricci flow, Perelman [5] has proposed an approach based on enlarging the space of coupling constants to include an extra function which then generates diffeomorphisms that act by pullback on $g_{i j}$. A choice of this function gives a submanifold of the enlarged space onto which the original space of coupling constants can be mapped, and can be thought of as a choice of parametrization of the coupling constants $g_{i j}$ in the sigma model. The first-order RG flow induces a flow on this submanifold, and the submanifold can be chosen so that the induced flow is gradient with respect to a positive definite metric. The submanifold is selected in a very natural way: one fixes the extra function above to be given by the lowest eigenfunction of a certain Schrödinger prob$\mathrm{lem}^{3}$ on the manifold $\left(M, g_{i j}\right)$. We have described this construction in greater detail in [7].

While Perelman's approach works to first order in $\alpha^{\prime}$, there remains the question of whether the full RG flow is gradient with respect to a positive definite metric. Tseytlin has recently addressed this question [4]. He starts with an action functional which is the integral over the target space of the "generalized central charge function," a particular combination of metric and dilaton $\beta$-functions discussed in $[8,9]$, to which he appends a Lagrange multiplier term. Upon truncating the generalized central charge to first order in $\alpha^{\prime}$ and extremizing the resulting action functional with respect to the dilaton, one can reproduce Perelman's construction, so the first-order RG flow of the target space metric is obtained as a gradient flow of the truncated action functional of Tseytlin. Then Tseytlin invokes results of Osborn [10] to argue that the untruncated gradient generates to all orders in perturbation theory the RG flow of the sigma model's target space metric. ${ }^{4}$

\footnotetext{
${ }^{3}$ A special case of this Schrödinger problem first appeared in the study of RG flows in [6], which studied the case of a 2dimensional target space.

${ }^{4}$ In the process, the dilaton becomes metric dependent (it in fact satisfies the equation of the lowest eigenfunction of a Schrödinger operator describing the wave function of a particle coupled to gravity via the curvature scalar). This dilaton no longer satisfies its own independent RG flow equation.
}

The corresponding metric on the space of coupling constants is not explicitly given beyond first order in [4] (to that order it is just the metric obtained from Perelman's construction [5,7]). Thus the issue of monotonicity of this action functional under RG flow beyond first order remains to be explored. Tseytlin argues that a strict monotonicity formula is not necessary. Rather, since the leading (Perelman) term in the derivative of the action along the flow is positive, failure of monotonicity indicates that higher-order terms become dominant. This suggests that perhaps the perturbation series for the $\beta$-functions will fail to converge whenever monotonicity of the action fails; conversely, monotonicity holds whenever perturbation theory makes sense. A motivation for this expectation is the fact that the central charge action is related to the Zamolodchikov $C$-function, and upon applying Perelman's construction, the hope is that it indeed behaves like a $C$-function, and is monotonic under RG flow to all orders.

It is difficult to test this since the full perturbation series is not known explicitly. However, we take a pragmatic view. Say the $\beta$-functions are known to some order $p$. Then the central charge action (plus Lagrange multiplier term) is also known at this order, and one can compute its derivative along the flow and check for monotonicity. This will reveal the circumstances $C$, if any, in which monotonicity may fail at order $p$. If $C$ is nonempty, one can then attempt to estimate whether the order $p$ truncation of the $\beta$-functions is valid or whether higher-order, neglected terms are, in circumstances $C$, comparable in size to the lower-order, untruncated terms. If so, the order $p$ truncation breaks down; i.e., the truncation should be extended. The view in [4] would be confirmed if such an extension either restores monotonicity or eventually points to a divergent perturbation series, but these are not the only possible outcomes. A reliable assessment would require greater knowledge of the perturbation series than is presently available.

The purpose of the present work is to confirm that the issue does arise, because the metric that emerges from the proposal in [4] is not order by order of definite sign; indeed, the issue will arise at second order in $\alpha^{\prime}$.

There are essentially two ways in which truncations at finite order and perturbation theory may become unreliable. Judging from the known terms in the perturbation series for $\beta$ (e.g., [11]), these are when either (i) curvatures become large $\left(\sim 1 / \alpha^{\prime}\right.$ or larger), or (ii) derivatives of curvatures become large. The problem can occur even when the curvature is small in magnitude, if some derivative of curvature is sufficiently large. ${ }^{5}$

\footnotetext{
${ }^{5}$ One may suggest that RG flow will smooth out the inhomogeneities that generate large derivatives. This is not always clear. Ricci flow, for example, does not always smooth out inhomogeneities.
} 
Let us now look more closely at the mechanism by which monotonicity might fail when passing from leading order in $\alpha^{\prime}$ to next order. If $S$ is the action and RG flow is its gradient flow, then schematically at least, along the flow we have

$$
\begin{gathered}
\frac{d S}{d t}=\kappa(\beta, \beta) \\
=\int_{M}\left[\kappa_{(0)}^{i j k l}\left(\beta_{i j}^{(1)} \beta_{k l}^{(1)}+\beta_{i j}^{(1)} \beta_{k l}^{(2)}+\beta_{i j}^{(2)} \beta_{k l}^{(1)}+\cdots\right)\right. \\
\left.+\kappa_{(1)}^{i j k l} \beta_{i j}^{(1)} \beta_{k l}^{(1)}+\kappa_{(1)}^{i j k l m n} \nabla_{m} \beta_{i j}^{(1)} \nabla_{n} \beta_{k l}^{(1)}+\cdots\right] d m .
\end{gathered}
$$

Here $\kappa(\cdot, \cdot)$ is the metric on the space of coupling constants, $d m$ is some measure, and $\beta$ represents the $\beta$-function for the target space metric. The subscript or the superscript in parentheses indicates the order in $\alpha^{\prime}$, so we keep only terms up to order $\alpha^{13}$ inclusive (the leading term being of order $\alpha^{2}$ ). On dimensional grounds, higher derivatives than those shown cannot occur at this order. Since truncation at leading order is just the case studied in [5], we see that $\kappa_{(0)}^{i j k l}$ is positive semidefinite. Monotonicity at next-to-leading order becomes a question of the signatures of the two $\kappa_{(1)}$ coefficients.

We will confirm by explicit variation of the second-order action that to second order in $\alpha^{\prime}$ the RG flow is the gradient flow of Tseytlin's action functional and that its flow derivative has the form (1.7) [with the diffeomorphismimproved $\beta$-function $\bar{\beta}_{i j}$, defined in (1.8), appearing in place of $\beta$ above]. Furthermore, $\kappa_{(1)}^{i j k l}=0$, but $\kappa_{(1)}^{i j k l m n}$ is nonpositive so $\kappa$ (truncated at order $\alpha^{\prime}$ ) is no longer positive semidefinite and so the RG flow, truncated at second order, does not have a monotonicity formula. This happens precisely in situation (ii) above; i.e., when first derivatives of the curvatures are as large as $\mathcal{O}\left(\mid\right.$ Riem $\left.\mid / \sqrt{\alpha^{\prime}}\right)$, and may signal a breakdown in perturbation theory. Interestingly, large and even arbitrarily large curvatures will not violate monotonicity at second order if the curvature is sufficiently homogeneous - even though for large enough curvatures the sigma model perturbation theory certainly breaks down.

We find in particular that on Ricci solitons the monotonicity formula holds for the second-order RG flow. Indeed, monotonicity holds at second order on a wider class of metrics than solitons, namely those with harmonic curvature operator. This condition is not preserved along the second-order flow, so monotonicity along a flow that begins at a metric with harmonic curvature can eventually break down at large enough $t$ along the flow.

We follow [4] for our definitions of $\beta$-functions. In particular, we choose local coordinates on $M$ so that the RG flow of the target space metric $g_{i j}$ and dilaton $\phi$ is expressed as

$$
\begin{aligned}
\frac{\partial g_{i j}}{\partial t} & =-\bar{\beta}_{i j}^{g} \\
& =-\alpha^{\prime}\left(R_{i j}+2 \nabla_{i} \nabla_{j} \phi\right)-\frac{\alpha^{\prime 2}}{2} R_{i k l m} R_{j}^{k l m}+\mathcal{O}\left(\alpha^{13}\right),
\end{aligned}
$$

$$
\begin{aligned}
\frac{\partial \phi}{\partial t}= & -\bar{\beta}^{\phi} \\
= & -c_{0}+\alpha^{\prime}\left(\frac{1}{2} \Delta \phi-|\nabla \phi|^{2}\right)-\frac{\alpha^{\prime 2}}{16} \mid \text { Riem }\left.\right|^{2} \\
& +\mathcal{O}\left(\alpha^{\prime 3}\right) .
\end{aligned}
$$

This paper is organized as follows. Section II reviews Tseytlin's proposal and Perelman's technique. Section III extends the analysis to second order in $\alpha^{\prime}$. Section IV shows that the second-order flow is gradient and contains the formula for the derivative along the flow of Tseytlin's action $\mathcal{S}$. Section V contains a brief discussion of metrics for which monotonicity does not break down. We reiterate that, throughout, all manifolds are closed Riemannian manifolds.

\section{TSEYTLIN'S PROPOSED POTENTIAL}

In this section, we review Tseytlin's proposal and the result of Perelman upon which it is based.

Consider the "central charge action" [8,9], modified by a Lagrange multiplier term:

$$
\begin{aligned}
S(g, \phi) & :=\int_{M} \tilde{\beta}^{\phi} e^{-2 \phi} d V+\lambda\left(\int_{M} e^{-2 \phi} d V-1\right), \\
\tilde{\beta}^{\phi}:=\bar{\beta}^{\phi}-\frac{1}{4} g^{i j} \bar{\beta}_{i j}^{g} & \\
= & c_{0}-\alpha^{\prime}\left(\Delta \phi-|\nabla \phi|^{2}+\frac{1}{4} R\right)-\frac{\alpha^{\prime 2}}{16}|\mathrm{Riem}|^{2} \\
& +\mathcal{O}\left(\alpha^{\prime 3}\right) .
\end{aligned}
$$

Tseytlin's proposal is that the RG flow for $g_{i j}$ is the gradient of the action ${ }^{6}$

$$
\mathcal{S}(g):=\hat{S}(g, \varphi),
$$

where

$$
\varphi=-\log \Phi
$$

and $\Phi$ solves the eigenvalue problem

$$
\alpha^{\prime}\left(\Delta-\frac{1}{4} R-\frac{\alpha^{\prime}}{16} \mid \text { Riem }^{2}+\mathcal{O}\left(\alpha^{\prime 2}\right)\right) \Phi=-\left(\lambda+c_{0}\right) \Phi,
$$

\footnotetext{
${ }^{6}$ The sign convention for the action is opposite that of Perelman, so the desired monotonicity property will be a monotone decrease.
} 


$$
1=\int_{M} \Phi^{2} d V \equiv \int_{M} e^{-2 \varphi} d V
$$

In the action $\lambda$ appears as a Lagrange multiplier, and $c_{0}$ is a free parameter. Note that $c_{0}+\lambda$ must be the lowest eigenvalue of the operator on the left-hand side of $(2.5){ }^{7}$ since by (2.4) $\Phi$ cannot have nodes; otherwise the logarithm would fail to be defined. The eigenvalue problem (2.4), (2.5), and (2.6) arises by extremizing the action $\hat{S}(g, \phi)$ with respect to $\phi$ and $\lambda$. The dilaton RG flow cannot be obtained as a gradient flow of (2.3) since the action $\mathcal{S}(g)$ is not a functional of $\phi$.

It is easily checked that $(2.2),(2.3),(2.4),(2.5)$, and (2.6) imply

$$
\tilde{\beta}^{\varphi}=-\lambda=\mathcal{S}(g) .
$$

where of course $\lambda$ depends nontrivially on $g$ due to (2.5).

An arbitrary one-parameter variation of the action (2.1) yields

$$
\begin{aligned}
\frac{d S}{d s}= & \int_{M}\left[-\frac{1}{4} \bar{\beta}_{i j} \frac{\partial g^{i j}}{\partial s}-\frac{1}{4} g^{i j} \frac{\partial \bar{\beta}_{i j}}{\partial s}-\frac{\partial \bar{\beta}^{\phi}}{\partial s}\right] e^{-2 \phi} d V \\
& +\int_{M}\left(\tilde{\beta}^{\phi}+\lambda\right) \frac{\partial}{\partial s}\left(e^{-2 \phi} d V\right) \\
& +\frac{\partial \lambda}{\partial s}\left(\int_{M} e^{-2 \phi} d V-1\right) .
\end{aligned}
$$

If we vary about the minimizer $\phi=\varphi$, then due to (2.6) and (2.7) the last two integrals contribute nothing. Thus (2.8) reduces to

$$
\frac{d S}{d s}=\int_{M}\left[-\frac{1}{4} \bar{\beta}_{i j} \frac{\partial g^{i j}}{\partial s}-\frac{1}{4} g^{i j} \frac{\partial \bar{\beta}_{i j}}{\partial s}-\frac{\partial \bar{\beta}^{\phi}}{\partial s}\right]_{\phi=\varphi} e^{-2 \varphi} d V .
$$

Section 1 of [5] (see also [7]) shows that if the $\beta$-functions are replaced by their first-order truncations (at the mini$\operatorname{mizer} \varphi$ )

$$
\begin{gathered}
\bar{\beta}_{i j}^{(1)}=\alpha^{\prime}\left(R_{i j}+2 \nabla_{i} \nabla_{j} \varphi\right), \\
\bar{\beta}^{\varphi(1)}=c_{0}-\alpha^{\prime}\left(\frac{1}{2} \Delta \varphi-|\Delta \varphi|^{2}\right),
\end{gathered}
$$

then the last two terms in the integrand vanish. One obtains simply

$$
\frac{d S^{(1)}}{d s}=\frac{1}{4} \int_{M} g^{i k} g^{j l} \bar{\beta}_{i j}^{(1)} \frac{\partial g_{k l}}{\partial s},
$$

so the first-order truncated flow

$$
\frac{\partial g_{i j}}{\partial t}=-\bar{\beta}_{i j}^{(1)}
$$

is clearly gradient, the metric is

$$
(u, v)=\int_{M} g^{i k} g^{j l} u_{i j} v_{k l},
$$

which is positive semidefinite, and along the flow we have the monotonicity formula

$$
\frac{d S^{(1)}}{d t}=-\frac{1}{4} \int_{M}\left|\bar{\beta}_{i j}^{(1)}\right|^{2} .
$$

This implies that the derivative (2.9) along the flow of the full action has the form

$$
\frac{d S}{d t}=-\frac{1}{4} \int_{M}\left(\left|\bar{\beta}_{i j}\right|^{2}+\mathcal{O}\left(\alpha^{13}\right)\right),
$$

where the $\left|\bar{\beta}_{i j}\right|^{2}$ term is $\mathcal{O}\left(\alpha^{\prime 2}\right)$.

\section{SECOND-ORDER ACTION}

In this section, we include in the action the term $\alpha^{\prime 2} \mid$ Riem $\left.\right|^{2}$ which occurs in $\tilde{\beta}^{\phi}$, and compute its variation. The result can also be reconstructed from calculations in the literature (see [11]). Readers wishing to skip the routine calculational details may want to proceed straight to the results (3.11) and (3.12).

For a one-parameter variation in the metric, where $s$ is the parameter, we use the standard formulas

$$
\begin{gathered}
\frac{\partial}{\partial s} R_{j k l}^{i}=\nabla_{k} \frac{\partial}{\partial s} \Gamma_{j l}^{i}-\nabla_{l} \frac{\partial}{\partial s} \Gamma_{j k}^{i}, \\
\frac{\partial}{\partial s} \Gamma_{j k}^{i}=\frac{1}{2} g^{i l}\left(\nabla_{j} \frac{\partial g_{l k}}{\partial s}+\nabla_{k} \frac{\partial g_{j l}}{\partial s}-\nabla_{l} \frac{\partial g_{j k}}{\partial s}\right), \\
\frac{\partial}{\partial s} d V=\frac{1}{2} g^{i j} \frac{\partial g_{i j}}{\partial s} d V .
\end{gathered}
$$

Using these, we write

$$
\begin{aligned}
\frac{\partial}{\partial s}\left[-\frac{\alpha^{\prime 2}}{16} \int_{M}|\operatorname{Riem}|^{2} e^{-2 \phi} d V\right]= & -\frac{\alpha^{\prime 2}}{16} \int_{M}\left[2 R^{p}{ }_{q r s} g_{p i} g^{q j} g^{r k} g^{s l} \frac{\partial}{\partial s} R_{j k l}^{i}+R_{k l m}^{i} R^{j k l m} \frac{\partial g_{i j}}{\partial s}+R_{k l m}^{i} R_{i j}{ }^{l m} \frac{\partial g^{j k}}{\partial s}\right. \\
& \left.+R_{k l m}^{i} R_{i}{ }_{j}{ }_{j} \frac{\partial g^{j l}}{\partial s}+R_{k l m}^{i} R_{i}{ }^{k l}{ }_{j} \frac{\partial g^{m j}}{\partial s}+|\operatorname{Riem}|^{2}\left(\frac{1}{2} g^{i j} \frac{\partial g_{i j}}{\partial s}-2 \frac{\partial \phi}{\partial s}\right)\right] e^{-2 \phi} d V \\
= & -\frac{\alpha^{\prime 2}}{16} \int_{M}\left[2 R^{i j k l} \nabla_{k}\left(\nabla_{j} \frac{\partial g_{i l}}{\partial s}+\nabla_{l} \frac{\partial g_{i j}}{\partial s}-\nabla_{i} \frac{\partial g_{j l}}{\partial s}\right)-2 R_{k l m}^{i} R^{j k l m} \frac{\partial g_{i j}}{\partial s}\right. \\
& \left.+|\operatorname{Riem}|^{2}\left(\frac{1}{2} g^{i j} \frac{\partial g_{i j}}{\partial s}-2 \frac{\partial \phi}{\partial s}\right)\right] e^{-2 \phi} d V .
\end{aligned}
$$

\footnotetext{
${ }^{7} \varphi$ is therefore sometimes called the minimizer.
} 
The term $R^{i j k l} \nabla_{k} \nabla_{l} \frac{\partial g_{i j}}{\partial s}$ is easily seen by index symmetry to contribute zero, so we will discard it. Next, we integrate by parts and use the second Bianchi identity, once contracted, which shows that

$$
\nabla_{k} R^{i j k l}=\nabla^{i} R^{j l}-\nabla^{j} R^{i l} .
$$

The result is

$$
\begin{aligned}
\frac{\partial}{\partial s}[- & \left.\frac{\alpha^{\prime 2}}{16} \int_{M} \mid \text { Riem }\left.\right|^{2} e^{-2 \phi} d V\right] \\
= & -\frac{\alpha^{\prime 2}}{16} \int_{M}\left[2\left(\nabla^{j} R^{i l}-\nabla^{i} R^{j l}\right)\left(\nabla_{j} \frac{\partial g_{i l}}{\partial s}-\nabla_{i} \frac{\partial g_{j l}}{\partial s}\right)\right. \\
& +4 R^{i j k l} \nabla_{k} \phi\left(\nabla_{j} \frac{\partial g_{i l}}{\partial s}-\nabla_{i} \frac{\partial g_{j l}}{\partial s}\right)-2 R_{k l m}^{i} R^{j k l m} \frac{\partial g_{i j}}{\partial s} \\
& \left.+|\operatorname{Riem}|^{2}\left(\frac{1}{2} g^{i j} \frac{\partial g_{i j}}{\partial s}-2 \frac{\partial \phi}{\partial s}\right)\right] e^{-2 \phi} d V .
\end{aligned}
$$

We can replace the $R^{i j k l} \nabla_{k} \phi$ term using the Ricci identity

$$
R^{i j k l} \nabla_{k} \phi=-\left(\nabla^{i} \nabla^{j}-\nabla^{j} \nabla^{i}\right) \nabla^{l} \phi .
$$

Finally, if we vary about the minimizer $\phi=\varphi$, then $\left(\frac{1}{2} g i j \frac{\partial g_{i j}}{\partial s}-2 \frac{\partial \phi}{\partial s}\right)$ vanishes. Using these results, we obtain

$$
\begin{aligned}
\frac{\partial}{\partial s}[ & \left.-\frac{\alpha^{\prime 2}}{16} \int_{M} \mid \mathrm{Riem}^{2} e^{-2 \phi} d V\right] \\
= & -\frac{\alpha^{\prime 2}}{16} \int_{M}\left\{2\left[\nabla^{j}\left(R^{i l}+2 \nabla^{i} \nabla^{l} \phi\right)-\nabla^{i}\left(R^{j l}+2 \nabla^{j} \nabla^{l} \phi\right)\right]\right. \\
& \left.\times\left(\nabla_{j} \frac{\partial g_{i l}}{\partial s}-\nabla_{i} \frac{\partial g_{j l}}{\partial s}\right)-2 R_{k l m}^{i} R^{j k l m} \frac{\partial g_{i j}}{\partial s}\right\} e^{-2 \phi} d V \\
= & \left.\frac{\alpha^{\prime}}{8} \int_{M}\left(\nabla^{j} \bar{\beta}^{(1) i l}-\nabla^{i} \bar{\beta}^{(1) j l}\right)\left(\nabla_{j} \frac{\partial g_{i l}}{\partial s}-\nabla_{i} \frac{\partial g_{j l}}{\partial s}\right)\right|_{\phi=\varphi} \\
& \times e^{2 \varphi} d V+\frac{1}{4} \int_{M} \bar{\beta}^{(2) i j} \frac{\partial g_{i j}}{\partial s} e^{-2 \varphi} d V,
\end{aligned}
$$

where we define

$$
\bar{\beta}_{i j}^{(2)}=\frac{\alpha^{\prime}}{2} R_{i k l m} R_{j}^{k l m}
$$

so that

$$
\bar{\beta}_{i j}=\bar{\beta}_{i j}^{(1)}+\bar{\beta}_{i j}^{(2)}+\mathcal{O}\left(\alpha^{\prime 3}\right) .
$$

Combining (2.12) and (3.8), we obtain

$$
\begin{aligned}
\frac{d \mathcal{S}}{d s}= & \int_{M}\left\{\frac{1}{4} \bar{\beta}^{i j} \frac{\partial g_{i j}}{\partial s}-\frac{\alpha^{\prime}}{8}\left[\nabla^{i} \bar{\beta}^{j k}-\nabla^{j} \bar{\beta}^{i k}\right]\right. \\
& \left.\times\left(\nabla_{i} \frac{\partial g_{j k}}{\partial s}-\nabla_{j} \frac{\partial g_{i k}}{\partial s}\right)+\left.\alpha^{\prime 3} T^{i j} \frac{\partial g_{i j}}{\partial s}\right|_{\varphi}\right\} e^{-2 \varphi} d V,
\end{aligned}
$$

where $T \in \mathcal{O}(1)$ is the coefficient of the error estimate. Lastly, integrating by parts, we can express this in the form

$$
\begin{aligned}
\frac{d \mathcal{S}}{d s}= & \int_{M} \frac{\partial g_{i j}}{\partial s} g^{j l}\left\{\frac{1}{4}\left[g^{i k}\left(1+\alpha^{\prime} \tilde{\Delta}\right)-\alpha^{\prime} \widetilde{\mathrm{Hess}}^{i k}\right] \bar{\beta}_{k l}\right. \\
& \left.+\alpha^{\prime 3} T_{l}^{i}\right\} e^{-2 \varphi} d V .
\end{aligned}
$$

Here $\widetilde{\operatorname{Hess}}^{i k}(\cdot):=e^{2 \varphi} \nabla^{k}\left(e^{-2 \varphi} \nabla^{i}(\cdot)\right)$ and $\tilde{\Delta}:=g_{i k} \widetilde{\operatorname{Hess}}^{i k}$.

\section{GRADIENT FLOW AND MONOTONICITY}

In the finite-dimensional case, the flow

$$
\frac{d x^{i}}{d t}=F^{i}
$$

generated by vector field $F^{i}$ is a gradient flow iff for a metric $\kappa$

$$
F^{i}=\kappa^{i k} \partial_{k} V
$$

That is, $F$ is the gradient vector arising from raising the index on the exterior derivative of a scalar potential $V$. Equivalently, $F$ must obey

$$
\partial_{i} F_{j}-\partial_{j} F_{i}=0, \quad F_{i}:=\kappa_{i k} F^{k} .
$$

The directional derivative of $V$ in the direction of an arbitrary tangent vector $v^{i}=d x^{i} / d s$ is of course just

$$
\frac{d V}{d s}=\frac{d x^{k}}{d s} \partial_{k} V
$$

In the infinite-dimensional case, the sums over $k$ become integrals, the directional derivative (4.2) becomes a variational derivative, and $\partial_{i} V$ becomes measure valued (i.e., a distribution in the sense of Dirac). Given a candidate potential function for a given flow generated by a known vector field $F$, one can perform the variational derivative to read off the analogue of $\partial_{k} V$ and then compare this to $F$ if the metric is known. (When taking the variational derivative, the vector field $d x^{i} / d s$ is replaced by the cotangent field $\partial g_{i j} / \partial s$.)

We claim that a suitable metric $\kappa(\cdot, \cdot)$ on the space of couplings is

$$
\begin{aligned}
\kappa(u, v):= & \frac{1}{4} \int_{M} d V e^{-2 \varphi}\left[g^{i k} g^{j l} u_{i j} v_{k l}\right. \\
& -\frac{\alpha^{\prime}}{2} g^{i k} g^{j l} g^{m n}\left(\nabla_{m} u_{i j}-\nabla_{i} u_{m j}\right) \\
& \left.\times\left(\nabla_{n} v_{k l}-\nabla_{k} v_{n l}\right)\right]
\end{aligned}
$$

\footnotetext{
${ }^{8}$ In other words, divergences are defined with respect to the measure $e^{-2 \varphi} d V$. At leading order in $\alpha^{\prime}$, which is all that we require here, this does not differ from the ordinary divergence which appears in the comparable results in Sec. 2 of [11].
} 


$$
\begin{aligned}
= & \int_{M} u_{i j} g^{j l}\left\{\frac{1}{4}\left[g^{i k}\left(1+\alpha^{\prime} \tilde{\Delta}\right)-\alpha^{\prime} \widetilde{\operatorname{Hess}}^{i k}\right] v_{k l}\right. \\
& \left.+\mathcal{O}\left(\alpha^{\prime 2}|v|\right)\right\} e^{-2 \varphi} d V,
\end{aligned}
$$

using integration by parts to obtain the last equality.

Compare (3.12) to (4.6). Assuming that $T^{i j}$ is linear in $\bar{\beta}_{i j}$ and using

$$
\frac{d \mathcal{S}}{d s}=-\kappa\left(\frac{\partial g}{\partial s}, \operatorname{Grad} \mathcal{S}\right),
$$

then we can read off that the gradient of $\mathcal{S}$ with respect to the metric $\kappa$ is

$$
\operatorname{Grad} \mathcal{S}=-\bar{\beta}_{i j}+\mathcal{O}\left(\alpha^{\prime}\right),
$$

establishing the claim.

For $u=v,(4.5)$ yields

$$
\kappa(u, u)=\frac{1}{4} \int_{M} d V e^{-2 \varphi}\left[\left|u_{i j}\right|^{2}-\frac{\alpha^{\prime}}{2}\left|\nabla_{i} u_{j k}-\nabla_{j} u_{i k}\right|^{2}\right] .
$$

Notice the overall minus sign in front of the gradient terms. Evaluating the derivative of $\mathcal{S}$ along a flow given by (1.8), the result (4.9) leads to

$$
\begin{aligned}
\frac{d \mathcal{S}}{d t}= & -\kappa(-\bar{\beta},-\bar{\beta}) \\
= & -\frac{1}{4} \int_{M} d V e^{-2 \varphi}\left[\left|\bar{\beta}_{i j}\right|^{2}-\frac{\alpha^{\prime}}{2}\left|\nabla_{i} \bar{\beta}_{j k}-\nabla_{j} \bar{\beta}_{i k}\right|^{2}\right. \\
& \left.+\mathcal{O}\left(\alpha^{\prime 4}\right)\right] .
\end{aligned}
$$

Thus as long as the nonderivative term dominates, $\mathcal{S}$ is monotonically decreasing along the RG flow.

\section{DISCUSSION}

The action $\mathcal{S}$ will fail to be monotonically decreasing at second order whenever

$$
\left|\nabla_{i} \bar{\beta}_{j k}-\nabla_{j} \bar{\beta}_{i k}\right|>\sqrt{\frac{2}{\alpha^{\prime}}}\left|\bar{\beta}_{i j}\right| .
$$

This situation is possible because we can always choose initial data for the flow with spatial gradients that obey $|\nabla \mathrm{Ric}| \sim|\mathrm{Ric}| / \sqrt{\alpha^{\prime}}$. Then both terms in the integrand of (4.10) are comparable in magnitude, and it may well be that the second term dominates, making $\mathcal{S}$ increase. However, when (5.1) holds, then $\mid \nabla$ Ric $|\sim| \operatorname{Ric} \mid / \sqrt{\alpha^{\prime}}$. Then the second-order truncation of the $\beta$-function is no longer reliable because third order terms are comparably large (cf. [11]). The second-order truncation breaks down. This scenario and its possible outcomes were described in the Introduction.

We turn now to circumstances for which monotonicity does hold, at least for an interval of "time" (energy scale).
One such class is the class of manifolds with harmonic curvature. These are precisely the metrics for which

$$
\nabla_{k} R^{i j k l} \equiv \nabla^{i} R^{j l}-\nabla^{j} R^{i l}=0 .
$$

Einstein manifolds obviously belong to this class, as do the Riemannian products of Einstein manifolds (as these have parallel Ricci tensor $\nabla_{i} R_{j k}=0$ ). Contracting this expression with $g^{j l}$ and using the contracted second Bianchi identity, we see that such manifolds must have constant scalar curvature. ${ }^{9}$ Then (2.5) and (2.6) admits solutions for $\Phi$ of the form $1+\mathcal{O}\left(\alpha^{\prime}\right)$ and then $\varphi \in \mathcal{O}\left(\alpha^{\prime}\right)$ (i.e., $\varphi^{(0)}=$ $0)$. It follows that

$$
\begin{aligned}
{\left[\nabla_{k} \bar{\beta}_{i j}^{g}-\nabla_{i} \bar{\beta}_{k j}^{g}\right] } & \equiv \alpha^{\prime}\left[\nabla_{k} R_{i j}-\nabla_{i} R_{k j}-R_{j k i}^{l} \nabla_{l} \varphi\right] \\
& \in \mathcal{O}\left(\alpha^{\prime 2}\right),
\end{aligned}
$$

assuming $\mathcal{O}(1)$ bounds on the curvature. Provided the solution is not "nearly solitonic" (i.e., provided $\left.\bar{\beta}^{(1)} \notin \mathcal{O}\left(\alpha^{\prime 2}\right)\right)$, then $d \mathcal{S} / d t<0$.

The condition of harmonic curvature cannot be expected to be preserved along the flow in general. If $t$ becomes large enough, an initially harmonic curvature can eventually deviate quite a bit from harmonicity.

A second class that obeys monotonicity is the class of gradient Ricci solitons, including so-called shrinkers and expanders as well as steadies. ${ }^{10}$ These obey

$$
\bar{\beta}_{i j}^{(1)} \equiv \alpha^{\prime}\left(R_{i j}+2 \nabla_{i} \nabla_{j} \varphi\right)=\alpha^{\prime} \lambda g_{i j},
$$

where $\lambda$ is a constant. Clearly, for this class, $\nabla_{i} \bar{\beta}_{j k}^{(1)}=0$, so the wrong-sign term in (4.10) vanishes, while the leading term integrates to give

$$
\frac{d \mathcal{S}}{d t}=-\frac{n \lambda^{2} \alpha^{12}}{4}+\mathcal{O}\left(\alpha^{13}\right)
$$

in dimension $n$, where $\mathcal{O}\left(\alpha^{13}\right)$ denotes the contribution from $\bar{\beta}_{i j}^{(2)}$. Again, this class will not be preserved along the flow, but deviations will be governed by the $\alpha^{2} R_{i k l m} R_{j}{ }^{k l m}$ term in (1.8), and such deviations, if absent initially, will not be important for quite some time. In fact, all that is required is that the evolving metric have gradient of $\bar{\beta}_{i j}$ close to that of a soliton metric; i.e., close to zero.

\section{ACKNOWLEDGMENTS}

We are grateful to Arkady Tseytlin for his comments on a draft of this paper. E.W. would like to thank the Department of Mathematics and Statistics, University of New Brunswick, for hospitality during the beginning of

\footnotetext{
${ }^{9}$ Harmonic curvature implies that the Bach tensor vanishes, and in any dimension $>3$ vanishing Bach tensor implies harmonic curvature. Quite a lot is known about these manifolds (see [12] and references therein).

${ }^{10}$ Since we work with compact manifolds, the steady solitons are all Ricci-flat [13].
} 
this work; the Albert Einstein Institute for an invitation to the Workshop on Geometric and Renormalization Group Flows, whose stimulating atmosphere led to the work's completion; and H.-P. Künzle for a discussion of manifolds of harmonic curvature. This work was partially supported by a grant from the Natural Sciences and Engineering Research Council of Canada.
[1] D. Friedan, Ann. Phys. (N.Y.) 163, 318 (1985).

[2] Gary Gibbons (unpublished).

[3] A. B. Zamolodchikov, JETP Lett. 43, 730 (1986).

[4] A. A. Tseytlin, Phys. Rev. D 75, 064024 (2007).

[5] G. Perelman, arXiv:math.DG/0211159.

[6] V. A. Fateev, E. Onofri, and A. B. Zamolodchikov, Nucl. Phys. B406, 521 (1993).

[7] T. Oliynyk, V. Suneeta, and E. Woolgar, Nucl. Phys. B739, 441 (2006).

[8] C. G. Callan, I. R. Klebanov, and M. J. Perry, Nucl. Phys. B278, 78 (1986).
[9] A. A. Tseytlin, Phys. Lett. B 194, 63 (1987).

[10] H. Osborn, Nucl. Phys. B294, 595 (1987); B308, 629 (1988); Ann. Phys. (N.Y.) 200, 1 (1990); Nucl. Phys. B363, 486 (1991).

[11] I. Jack, D. R. T. Jones, and N. Mohammedi, Nucl. Phys. B322, 431 (1989).

[12] A. L. Besse, Einstein Manifolds (Springer, Berlin, 1987), Chap. 16.E, pp. 443-447.

[13] J.-P. Bourguignon, in Global Differential Geometry and Global Analysis, Lectures in Mathematics Vol. 838, edited by D. Ferus (Springer, Berlin, 1981). 